\title{
An exploration of the determinants for decision to migrate existing resources to cloud computing using an integrated TOE- DOI model
}

\author{
Adel Alkhalil ${ }^{1 *}$ (D), Reza Sahandi ${ }^{2}$ and David John ${ }^{2}$
}

\begin{abstract}
Migrating existing resources to cloud computing is a strategic organisational decision that can be difficult. It requires the consideration and evaluation of a wide range of technical and organisational aspects. Although a significant amount of attention has been paid by many industrialists and academics to aid migration decisions, the procedure remains difficult. This is mainly due to underestimation of the range of factors and characteristics affecting the decision for cloud migration. Further research is needed to investigate the level of effect these factors have on migration decisions and the overall complexity. This paper aims to explore the level of complexity of the decision to migrate the cloud. A research model based on the diffusion of innovation (DOI) theory and the technology-organization-environment (TOE) framework was developed. The model was tested using exploratory and confirmatory factor analysis. The quantitative analysis shows the level of impact of the identified variables on the decision to migrate. Seven determinants that contribute to the complexity of the decisions are identified. They need to be taken into account to ensure successful migration. This result has expanded the collective knowledge about the complexity of the issues that have to be considered when making decisions to migrate to the cloud. It contributes to the literature that addresses the complex and multidimensional nature of migrating to the cloud.
\end{abstract}

Keywords: Cloud computing, Cloud migration, Decision-making process, Cloud migration factors, Cloud migration complexity, TOE, DOI

\section{Introduction}

Over the last decade, advances in computing have enabled cost-effective realisation of large-scale data centres [1]. It has led to computing being transformed to a model comprising services that are commoditised and delivered in a manner similar to traditional public utilities which stimulates a new paradigm - Cloud Computing. The emergence of this phenomenon fundamentally changed the way information systems are developed, deployed, scaled, supported, and paid for [2]. It has attracted many companies to migrate existing systems due to their promised advantages, particularly

\footnotetext{
*Correspondence: a.alkalel@uoh.edu.sa

${ }^{1}$ Faculty of Computer Science and Engineering, University of Hail, Hail, Saudi Arabia

Full list of author information is available at the end of the article
}

the reduction of capital expenses and the virtually infinite resource capacity that enhances businesses' agility [3]. Although cloud computing promises great benefits, it has not yet reached the maturity level that allows computing resources to be treated like commodities such as electricity as predicted by Buyya et al. [4]. Organisations are still reluctant to migrate their IT systems and services to the cloud due to concerns of losing control and other undesired outcomes [5]. Further, organisations are typically interested in moving only some of their systems to the cloud due to the difficulty of migrating the related applications, for example, safety-critical software [6]. Unlike start-up companies that develop systems from scratch, organisations planning to migrate existing legacy services to cloud computing often need the new services to be inter- 
operated with the existing systems [7]. This requires careful planning to avoid integration problem issues.

Being an important area for IT and business innovation, the adoption of cloud computing has received increasing attention in both practice and research [8]. Cloud computing is a fairly new provisioning model and a trend that involves continuing development of next-generation architecture. Therefore, most of the existing cloud studies are exploratory, descriptive, or case-based research [9]. For example, studies from [10-12] focus on the general conceptualization and definition of cloud computing, as well as strengths, weaknesses, opportunities and threats to the cloud computing industry. Further, many previous studies explored the business benefits and barriers for adoption. They focused mostly on the cost benefits, scalability, agility and the security issues, for examples see [13-17]. While these studies identified some factors that influence the decisions to migrate to the cloud, they were developed at an early stage of the evolvement of cloud computing. Further, the majority of them explored the factors affecting the adoption of cloud services while little attention was paid to the migration of legacy systems.

Many models and frameworks have been proposed to aid organisations with their decision to migrate to the cloud environment (e.g. [18-23]). Although significant contributions have been made by these works, there are still many aspects which require further support [24]. The main limitation in the proposed approaches is the separation of elements in the migration processes, that are connected and dependent on each other. Further, the existing support is usually limited to the selection of service providers such as [22] or to the adaption required for migrating applications such as [6]. Although evaluation of providers and their appropriate selection are critical, making an informed decision to migrate requires the analysis of a wide range of factors at early stages of the decision process. While the existing literature provides a fundamental understanding of cloud computing architecture, benefits and some issues, models and frameworks to support the migration and managing cloud services, research on drivers and barriers of the broad organizational adoption is still in early stages [5] and [9]. Therefore, this paper explores the issues that have increased the difficulties in organisations' decisions to migrate to the cloud. A two-stage survey was implemented for the exploration. This resulted in the identification of seven factors that contributed to the complexity of the decisions for migration. The analysis of the literature with regards to supporting the decision making process of companies when considering whether to migrate to the cloud shows that only some of those factors had been taken into account. The objective of this paper is to advance this further by empirically exploring the factors influencing the decision to migrate to the cloud. It proposes an integrated research model based on the Technology-Organization-Environment (TOE) framework and the Diffusion of Innovation theory (DOI).

\section{Theoretical background Migration to cloud computing}

Migration to the cloud, for the purpose of this paper, can be defined as the transition process of all or part of an organisation's legacy IT resources, including: hardware, software, stored data, and business processes, from locally on-premises deployments behind its firewalls to the cloud environment where they can be managed remotely by a third party. The process also encompasses the shifting of IT resources between different cloud providers, which is known as cloud-to-cloud migration. The cloud migration process may involve retaining some IT infrastructure on-site [25].

\section{Decision making process}

Simon [26] developed a generic decision making process model. The model is divided into three major phases: Intelligence, Design, and Choice. The process starts with the intelligence phase in which the 'reality' of an organisation is examined. It involves problem identification and information gathering activities about the societal, the competitiveness, and the organisational environments. The design phase simplifies an organisation's "reality" and identifies relationships between variables, as well as setting the criteria for evaluating alternative courses of action. The choice phase is to select the most appropriate alternative course of action based on the criteria identified in the design phase. The model has been widely accepted and adopted for problem-solving [27]. Turban et al. [28] described the model as the most concise, yet complete characterisation of rational decision making. Decision Support Systems (DSSs) are an integral part of Simon's model. The following section presents an analysis of the exiting DSSs, designed to support the decision making process for migrating to cloud computing. We applied Simons' [26] model on the existing DSSs to examine the level of support they offer in each of the three levels.

\section{Current level of support for decisions to migrate to the cloud}

The evolution and also the increase in popularity of cloud computing has led to a significant raise and awareness of industrialists and academics for the support required for migration decisions. Cloud vendors and IT consultancy agencies have made many attempts to provide guidance and assessment tools to help decisions for migration, as evidenced by a number of published whitepapers (See for example, [29-31]). However, these 
attempts have either been developed for marketing purposes or are not publicly available, due to them being based on closed proprietary technologies that usually require consultancy contracts [32]. Nevertheless, a number of DSSs have been proposed to support migration, as illustrated in Table 1.

A review of the DSSs shows that the vast majority of them do not support the assessment of the current cloud environments and business processes. In other words, they focus on supporting migration at the choice level for selecting providers. Although evaluation of providers and their appropriate selection are critical, making an informed decision to migrate requires the analysis of a wide range of factors at early stages of a decision process. Companies should develop a good awareness of the cloud environment and its capabilities, regulations, potentials and threats, before coming to a decision. Almost none of the reviewed work studied so far had considered "the intelligence level" and only a few paid attention to "the design level" for decision-making by considering the need for services adaptations in order to make them cloud enabled. For example, Andrikopoulos et al. [6] considered the adaptation required to the applications for migrating to the cloud.

The review also shows a high level of interest for migration for the IaaS model followed by the SaaS while there is very limited attention to the PaaS. Additionally, the existing approaches focus on migration from onpremises to the cloud while there is a lack of interest for migration from one provider to another. This is an important aspect to be addressed to avoid the issue of vendor lock-in which is a concern for many [33].

Further, the majority of the existing DSSs are prototypebased, experimental or conceptual. Therefore, the provision of established DSSs to include relevant information can substantially aid the decision making process. Another problem is the dispersed information, which is required for decision making. Although the information is available, it is often time consuming to bring all the details together. Additionally, due to the development of the technology and the expansion of the services offered, the range of information that is required to be considered for migration is increasing. Further, the availability of a wide range of DSSs and tools may create uncertainty for decision makers, if they are used outside of a systematic process.

Table $1 \mathrm{~A}$ review of the existing cloud DSSs

\begin{tabular}{|c|c|c|c|c|}
\hline Proposed approach & Cloud service & Factors taken into account & Method & Level of support \\
\hline $\begin{array}{l}\text { Suitability analysis for cloud } \\
\text { computing [45] }\end{array}$ & Not specified & $\begin{array}{l}\text { Size of the IT resources, the } \\
\text { utilisation pattern of the resources, } \\
\text { sensitivity of the data, and criticality } \\
\text { of the service }\end{array}$ & ROI model & Design \\
\hline CloudMIG [65] & PaaS and laaS & Applications reengineering & $\begin{array}{l}\text { Mathematical } \\
\text { modelling }\end{array}$ & Design and Choice \\
\hline Cloud adoption toolkit [18] & laaS & $\begin{array}{l}\text { Cost, characteristic social factors, } \\
\text { political factors, performance, } \\
\text { and practicalities }\end{array}$ & UML & Choice \\
\hline $\begin{array}{l}\text { DSS for migrating } \\
\text { applications [21] }\end{array}$ & SaaS & $\begin{array}{l}\text { Applications distribution, cloud } \\
\text { providers selection, elasticity strategy, } \\
\text { multi-tenancy requirements. }\end{array}$ & $\begin{array}{l}\text { Three-tiered } \\
\text { architecture }\end{array}$ & Design and Choice \\
\hline $\begin{array}{l}\text { DSS for migrating } \\
\text { applications [66] }\end{array}$ & SaaS & Cost and providers' characteristic & Conceptual modelling & Choice \\
\hline $\begin{array}{l}\text { Applications adaptations for } \\
\text { the cloud environment [6] }\end{array}$ & SaaS & The need for adaptation & Holistic approach & Design \\
\hline $\begin{array}{l}\text { partially migration of } \\
\text { applications to the cloud [67] }\end{array}$ & SaaS & Hybrid deployment & $\begin{array}{l}\text { Component } \\
\text { placement a and AHP }\end{array}$ & Design and Choice \\
\hline SMICloud [22] & Not specified & $\begin{array}{l}\text { Accountability, agility, assurance, } \\
\text { cost, performance, and security } \\
\text { and privacy. }\end{array}$ & $\begin{array}{l}\text { Component } \\
\text { placement a and AHP }\end{array}$ & Choice \\
\hline InCLOUDer [50] & SaaS & $\begin{array}{l}\text { Applications adaptations and } \\
\text { Accountability, agility, assurance, } \\
\text { cost, performance, and security } \\
\text { and privacy. }\end{array}$ & $\mathrm{AHP}$ & Design and Choice \\
\hline $\begin{array}{l}\text { DSS for migrating applications } \\
\text { [68] }\end{array}$ & SaaS and PaaS & The database layer of an application & $\begin{array}{l}\text { Step-by-step } \\
\text { methodology }\end{array}$ & Design \\
\hline CloudGenius [19] & laaS & $\begin{array}{l}\text { Cost, Performance, providers' } \\
\text { characteristic }\end{array}$ & $\begin{array}{l}\text { AHP and } \\
\text { mathematical } \\
\text { modelling }\end{array}$ & Choice \\
\hline Configuration support [23] & laaS & Cost and providers' characteristic & Feature model & Choice \\
\hline $\begin{array}{l}\text { Workflow Infrastructure } \\
\text { migration [56] }\end{array}$ & laaS & Cost and providers' characteristic & OPAL Simulation & Choice \\
\hline
\end{tabular}




\section{Organisational adoption of innovation}

The theoretical foundation for the exploration in this study is based on the TOE framework and DOI theory developed by Tornatzky and Fleischer [34] and Rogers [35] respectively, which are discussed below.

\section{The Technology-organisation-environment framework}

Although several frameworks have been developed for the adoption of ICT, the TOE is argued to be an integrative framework that provides a holistic approach and guidance [36]. The TOE framework consists of three dimensions of technology, organisation and environment that influence the process by which innovations are adopted (see Fig. 1). It serves as taxonomy for factors that facilitate or inhibit the adoption of technology innovations [37]. The framework has been used in the context of cloud commuting adoption as described by $[17,38,39]$.

The technological dimension relates to what is available to an organization and focuses on how a certain technology influences the adoption process [34]. The organisational context looks at the structure and the processes in an organization that constrain or facilitate the adoption and implementation of innovations [34]. The external environmental context is also supported by Tornatzky and Fleischer [34] such as the industry, competitors, regulations, and relationships with governments.

\section{The diffusion of innovation model}

The research on adoption of innovation continued in order to provide richer and possibly more explanatory models
[40]. A major contribution in this regard was the development of the Diffusion of Innovation model [35]. It has been widely used to explain IT adoptions. The model is concerned with the way that a new technological innovation progresses from creation to utilisation (see Fig. 2). It describes the patterns of adoption and the mechanisms for diffusion, as well as assisting to predict whether and how a new invention will be successful [35]. It has three main categories of factors that influence decisions to adopt innovations: Innovation Characteristics, Organizational Characteristics, and Individuals Characteristics. The innovation characteristics comprise the perceived attributes of the innovation that either encourage or hinder. Rogers [35] indicated that the five attributes of an innovation are: relative advantage, compatibility, complexity, trialability, and observability. Relative advantage refers to the level to which an advantage is perceived as better than the current system. Compatibility is the degree to which an innovation is perceived as being consistent with the existing values, past experiences and needs of potential adopters. Complexity relates to the perceived difficulty of understanding and using the innovation while trialability refers to the degree to which the innovation can be easily tried and tested over time. Finally, observability refers to the level to which the results of an innovation are visible to the technology adopter [35].

\section{Research design}

In order to answer the research questions, which focused on the exploration and support required for decision making for organisational migration to the cloud, a

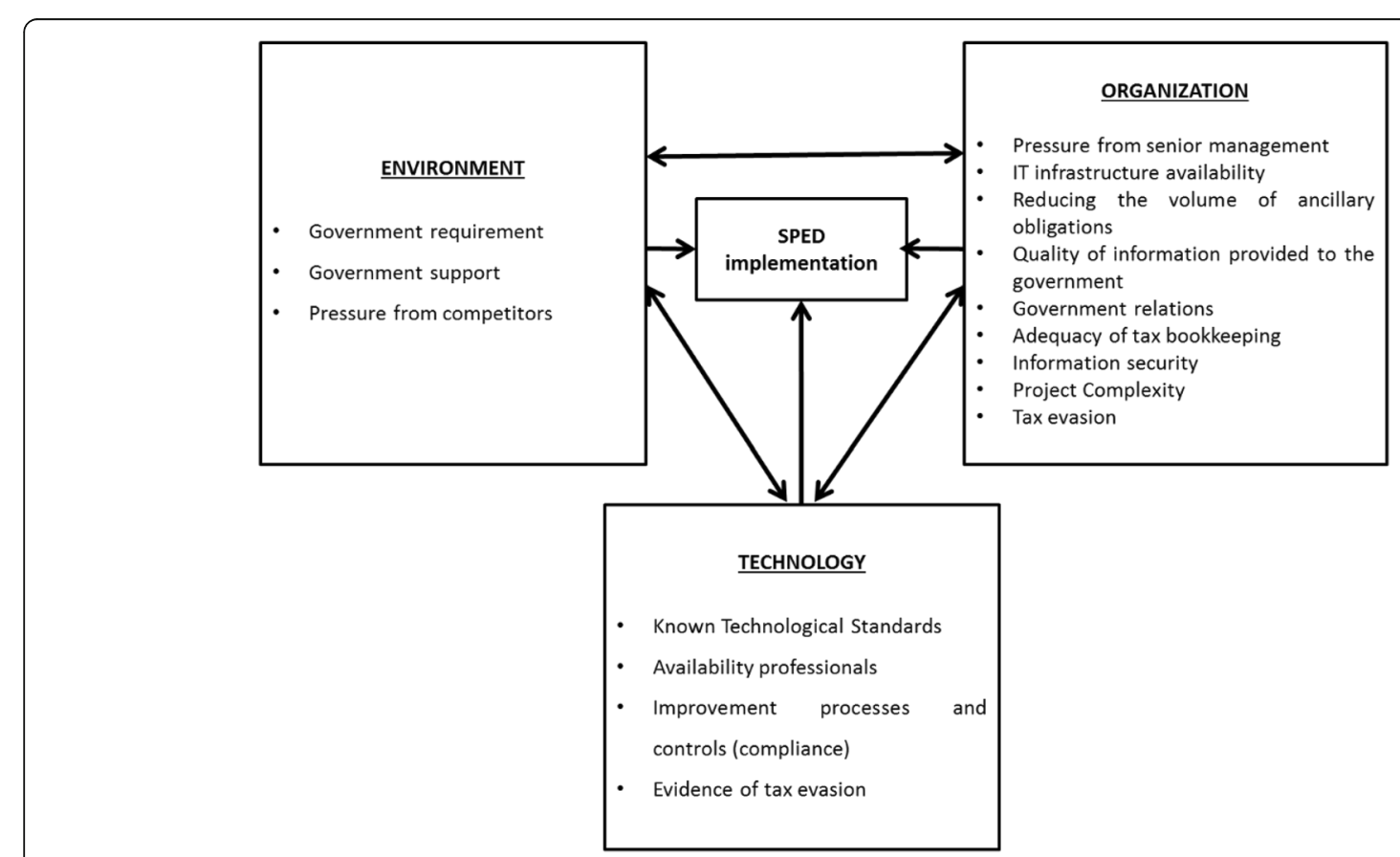

Fig. 1 The Technology-organisation-environment framework, Adapted from Tornatzky and Fleischer [34] 


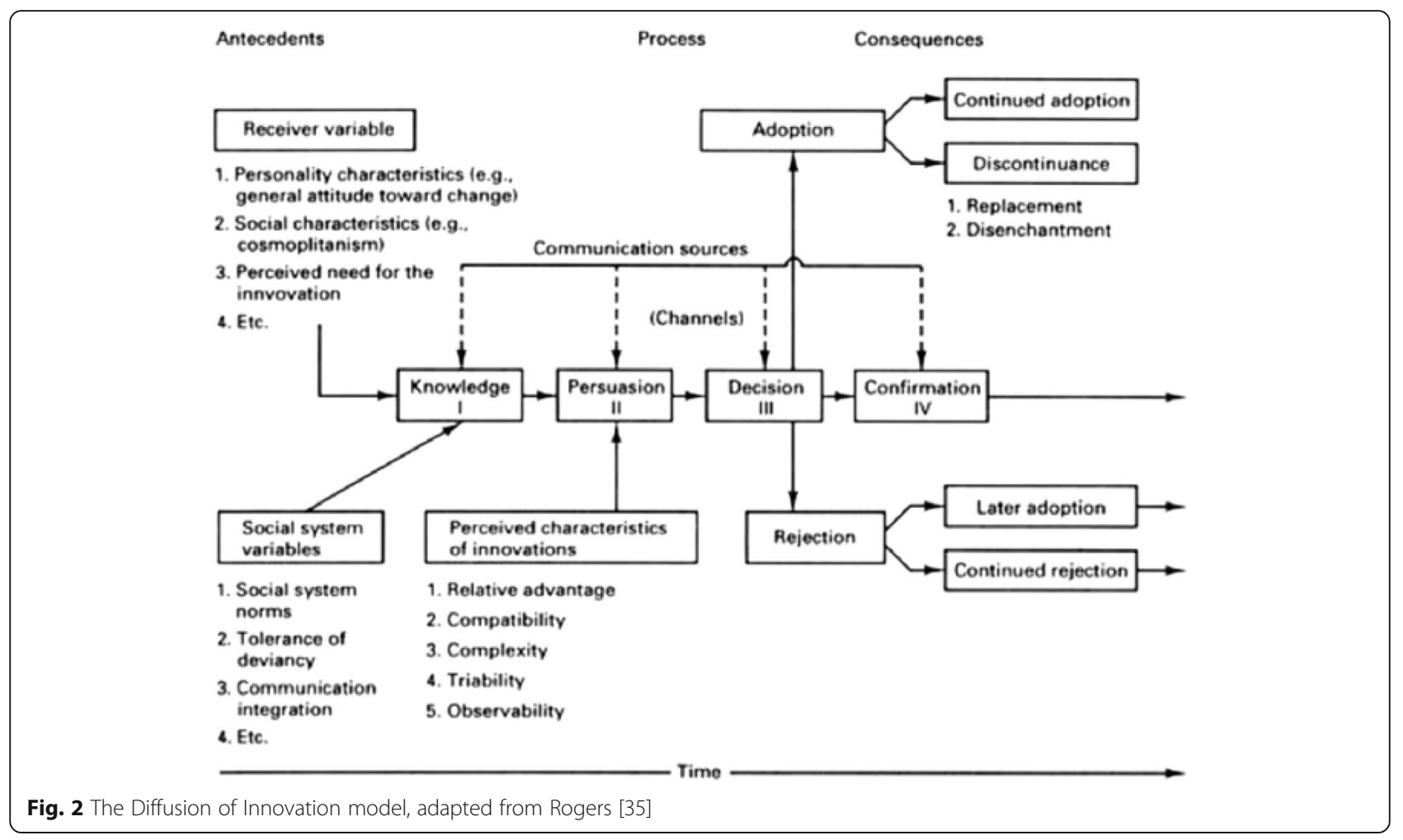

literature review was carried out. A number of factors and characteristics that increase the complexity of decisions to migrate were explored. The findings of the literature were supplemented with the implementation of a two-stage survey. The nature of the phenomenon studied in this research is comparatively new and is still evolving. Therefore, a sequential exploratory strategy was found to be the most appropriate way for gathering the needed data to answer the research questions. This strategy was tested using a two-stage survey that firstly gathered qualitative and then quantitative data (see Fig. 3).

Stage 1 was based on semi-structured interviews. Open ended questions were employed to ensure consistency, while it still allowed a degree of freedom and adaptability in obtaining the information from the interviewees. The interviews were conducted face-toface and on average each lasted about an hour. There were 12 interviewees in total who included IT managers (4), security professionals (3), and technical leaders within cloud provider companies (5) who were selected based on their subject expertise. The choice of the interview technique was based on the belief that real life practitioners, in particular service providers can offer a richer understanding of the benefits and challenges for adopting cloud computing due to their related experience. The interviews were carried out to further gain insight into the factors, issues, and concerns about the migration decisions, as well as developing a foundation for further analysis. The interviewees included decision makers of organisations in respect of cloud services, as well as some security experts. Twelve interviewees is a relatively small number; however, the sample mainly targeted technical professionals who were working for cloud provider companies. The information they provided was based on their experience of dealing with large number of customers, and during the interviews, they used examples of migration of some projects which raised the quality and reliability of the information they were able to share. Based on the analysis of stage 1 , as well as related literature, the research approach and hypotheses were formulated. In order to test the hypotheses, the stage 2 survey was implemented using an online survey questionnaire. Figure 3 shows the methodology adopted for this research.

\section{Data analysis (the exploration phase)}

Thematic analysis was used to analyse the qualitative data which was applied in six phases as suggested by [41]. The qualitative analysis enabled insights into challenges, issues, and factors that influence on the decision making process of whether to migrate to the cloud. To further specify these factors, the DOI and TOE frameworks were applied to the data. This resulted in findings within the following contexts: innovation characteristics, technology, organisation, and environment as shown in Table 2. The innovation characteristics was divided into four categories: relative advantages, compatibility, trialabilty, and probable risks. Participants indicated a number of advantages that can positively influence the decision to migrate to the cloud. They mostly agreed on cost 


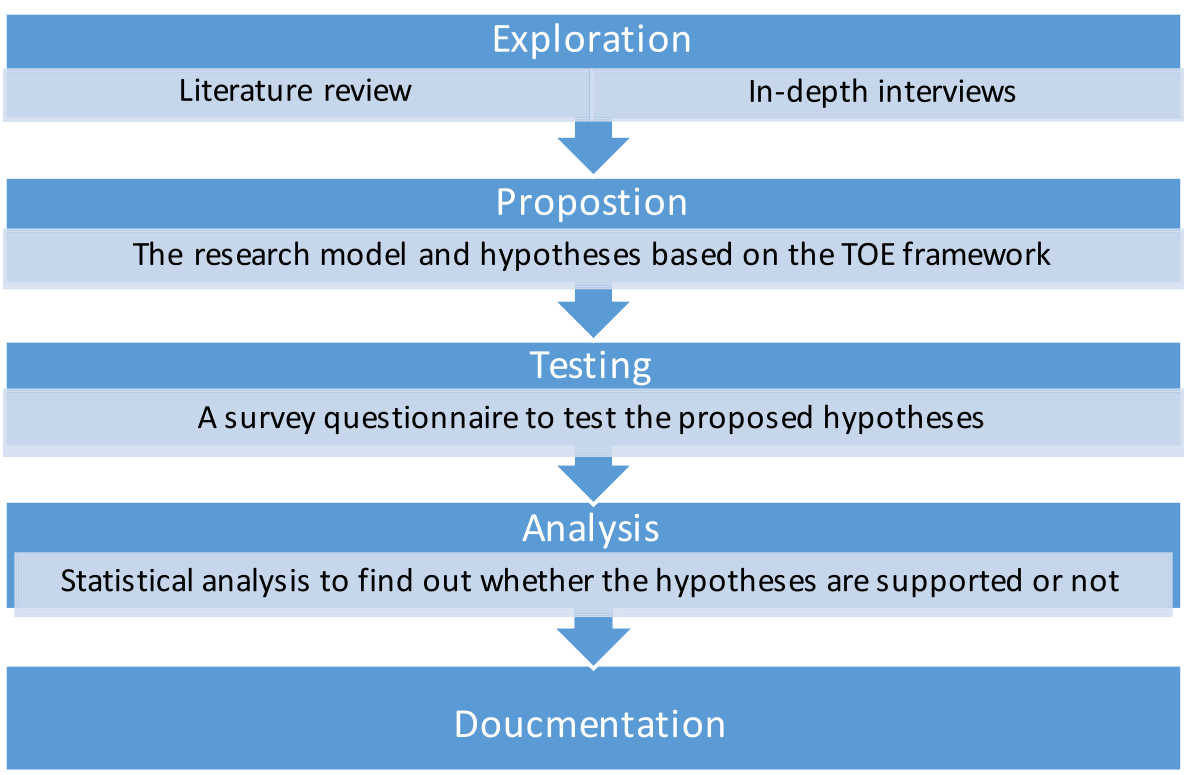

Fig. 3 Research design

reduction (91.6\%) and agility (75\%) followed by back-up (41.6\%) and higher performance (41.6\%) as positive drivers for migration. Further, two-thirds of the participants highlighted, testing advantages provided by the cloud as a positive driver for migration. On the other hand, participants reported a number of factors that increase risks and complexity (See Table 2). Lack of knowledge about the cloud environment was pointed out by all the cloud service providers interviewed in this study as a major issue that negatively influence the decision to migrate. Further, almost all the interviewees indicated there is a high level of concern for privacy and confidentiality regarding the adoption of cloud-based services. Within the technology context, participants indicated issues with compatibility and the difficulties of migrating large volume of legacy data as a negative influence on migration decisions. The impact on organisational culture, staff and interpretability issues were pointed out by $58 \%$ and the difficulties in

Table 2 The findings of stage 1 in the context of DOI and TOE

\begin{tabular}{|c|c|c|c|}
\hline Context & Variable & Findings & Impact \\
\hline Innovation & $\begin{array}{l}\text { Relative advantages } \\
\text { (DOI) }\end{array}$ & Cost reduction, agility, back-up, and higher performance & $\overline{\text { Positive }}$ \\
\hline \multirow[t]{3}{*}{ Characteristics } & Complexity (DOI) & $\begin{array}{l}\text { The lack of knowledge about the cloud environment, lack of skills } \\
\text { for managing cloud-service, cost management problems, risks } \\
\text { management, the immaturity of the cloud }\end{array}$ & Negative \\
\hline & Triability (DOI) & Ease of testing & Positive \\
\hline & Risks (DOI) & $\begin{array}{l}\text { Concerns of privacy and confidentiality, vendor lock in, and } \\
\text { loss of control }\end{array}$ & Negative \\
\hline \multirow[t]{2}{*}{ Technology } & Compatibility (DOI) & Impact on organisational culture and staff, interpretability issues & Negative \\
\hline & Size (TOE) & Difficulties in migrating large data & Negative \\
\hline \multirow[t]{4}{*}{ Organisation } & Organisation readiness (TOE) & Level of expertise & Negative \\
\hline & Internal social (TOE) & Need for adaptation, disruption to current business processes & Negative \\
\hline & External social (DOI) & Collaboration & Positive \\
\hline & $\begin{array}{l}\text { Top management } \\
\text { support (DOI) }\end{array}$ & Competitiveness, outsourcing culture, trust & Positive \\
\hline \multirow[t]{3}{*}{ Environment } & Information sources (TOE) & Difficult access to information and complexity & Negative \\
\hline & Regulation (TOE) & Concerns of legal implication, data ownership, and SLA & Negative \\
\hline & $\begin{array}{l}\text { Selection of cloud } \\
\text { provider (TOE) }\end{array}$ & $\begin{array}{l}\text { Selection of cloud services and providers is difficult, and Increasing } \\
\text { number of cloud provider and their configuration }\end{array}$ & Negative \\
\hline
\end{tabular}


migrating large data were pointed out by half of participants. The organisational context was divided into four variables (see Table 2) in which two were viewed as negative and the other two were viewed as positive. The level of expertise was pointed out by two-thirds of participants as a negative factor of organisations readiness to migrate. The need for adaptation and disruption to current business processes were considered as negative internal factors by $66.6 \%$ and $50 \%$ of the interviewees respectively. Collaboration and top management support were considered by half of the interviewees as positive factors encouraging for migration. In the context of environment, participants pointed out three elements that negatively influence the decision to migrate. Concerns about regulation were indicated by $75 \%$ followed by difficulty of access to information and complexity (58\%), and selection of cloud providers $(50 \%)$. Further analysis of the interviews can be found in [42] and [43]. Table 2 shows the main findings of stage 1 in the context of the DOI and TOE.

\section{Proposed model and hypotheses}

In this paper a model for identifying the determinants influencing the decision for cloud migration was developed (See Fig. 4). It is based on the integration of the characteristics contexts (organisation, innovation, individual, technology and environment) identified in the DOI and TOE frameworks. Combining more than one theoretical perspective was argued to enhance the understanding of adoption of innovative new technologies [44]. TOE and DOI have been widely accepted and adopted in IT adoption of innovation. They share some similarities, for example the consideration of technology and organisation context are almost the same in TOE and DOI. However, there are some differences between the two frameworks. DOI does not consider the environment context while in TOE, it is considered as part of organisational and technological context. In contrast, TOE does not consider individuals and some of the innovation characteristics, while DOI includes top management support in the organisational context and a wider range of the innovation characteristics. Generally, the TOE framework helps in identifying relevant categories for determinants while the DOI model helps in identifying specific variables within each category. Therefore, combining the two frameworks will complement each other and provide better understanding for the adoption. Further, in [41] it is argued that variables should be tailored to the characteristics of innovation. Therefore, the selection of variables is tailored to the context of migration to cloud computing. The variables were identified based on the analysis of the interviews in Stage 1, as well as the related literature. A hypothesis is created for each of the variables identified in the model, which are discussed in the following sections (Table 3 provides the list of the hypotheses).

\section{Innovation characteristics}

Relative advantages Migration to cloud computing can be a strategic decision for organisations to enhance the development of existing systems through improving

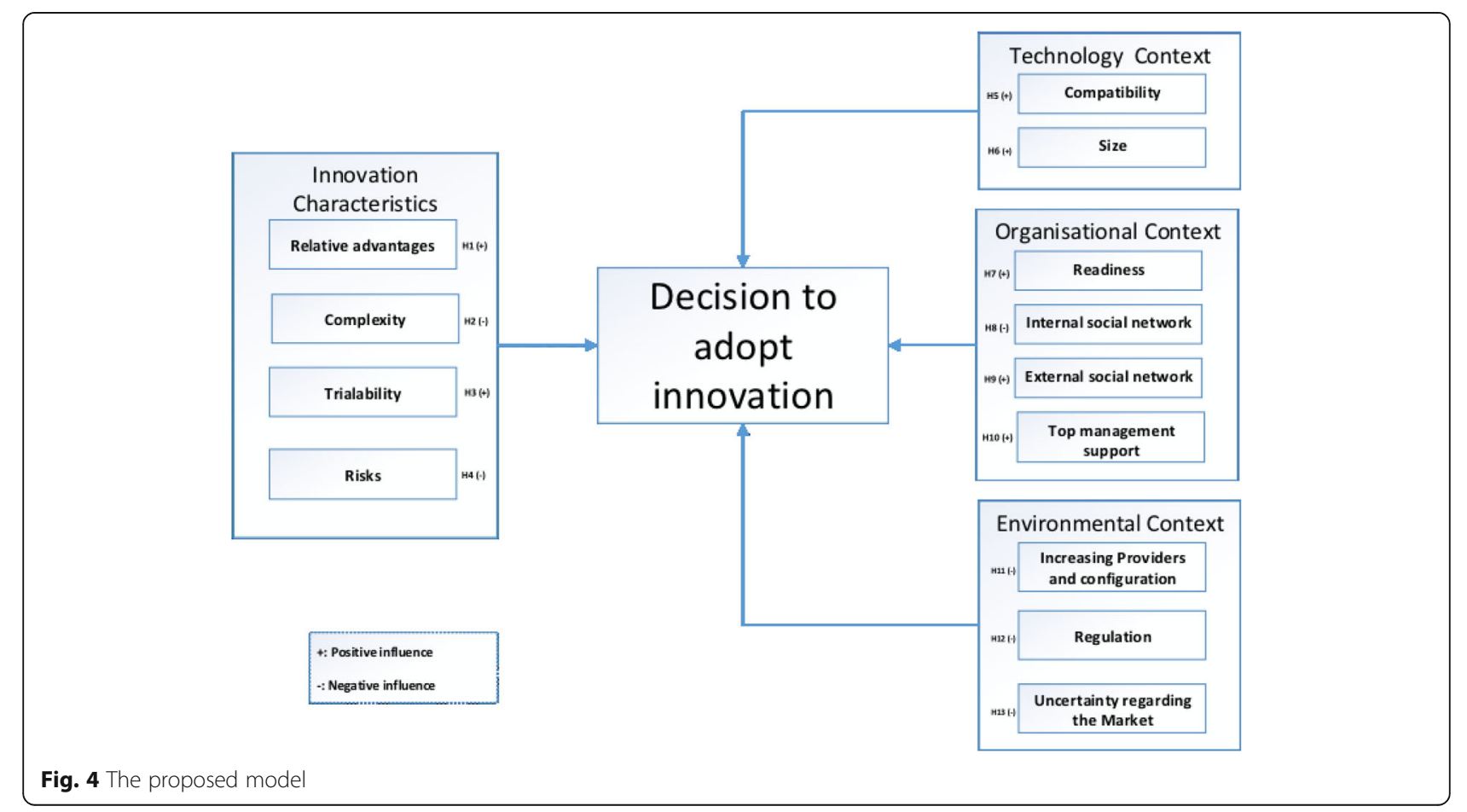


Table 3 List of hypotheses

\begin{tabular}{|c|c|}
\hline No & Hypotheses \\
\hline $\mathrm{H} 1$ & $\begin{array}{l}\text { Organisations that perceive high relative advantages of cloud } \\
\text { computing are more likely to migrate }\end{array}$ \\
\hline $\mathrm{H} 2$ & $\begin{array}{l}\text { Perceiving cloud computing as a complex technology will } \\
\text { negatively affect the decision to migrate }\end{array}$ \\
\hline $\mathrm{H} 3$ & $\begin{array}{l}\text { Ease of testing in the cloud will positively influence the } \\
\text { decision to migrate }\end{array}$ \\
\hline $\mathrm{H} 4$ & $\begin{array}{l}\text { High perception of risks will negatively influence the decision } \\
\text { to migrate }\end{array}$ \\
\hline H5 & $\begin{array}{l}\text { The perception that cloud computing is less compatible with } \\
\text { existing systems will negatively affect the decision to migrate }\end{array}$ \\
\hline H6 & High volumes of data are less likely to be migrated \\
\hline $\mathrm{H} 7$ & $\begin{array}{l}\text { Organisations readiness will positively influence the decision } \\
\text { to migrate to the cloud }\end{array}$ \\
\hline $\mathrm{H} 8$ & $\begin{array}{l}\text { The impact of migration on the internal social network will } \\
\text { negatively influence the decision to migrate }\end{array}$ \\
\hline H9 & $\begin{array}{l}\text { The impact of migration on external social network will } \\
\text { positively influence the decision to migrate }\end{array}$ \\
\hline $\mathrm{H} 10$ & $\begin{array}{l}\text { Upper management support is positively related to the decision } \\
\text { to migrate }\end{array}$ \\
\hline $\mathrm{H} 11$ & $\begin{array}{l}\text { The process of selecting a cloud provider is difficult which } \\
\text { negatively influences the decision to migrate }\end{array}$ \\
\hline $\mathrm{H} 12$ & $\begin{array}{l}\text { Concerns about legal implication are negatively related to } \\
\text { the decision to migrate }\end{array}$ \\
\hline $\mathrm{H} 13$ & $\begin{array}{l}\text { Difficulties of information gathering will negatively influence } \\
\text { the decision to migrate }\end{array}$ \\
\hline
\end{tabular}

scalability, flexibility, and time to market. The analysis of Stage 1 shows that cost reduction appears to be the strongest motivating factor from the participants' perspective for migration to cloud computing followed by agility. IT managers who participated in the survey believe that the need to implement a new service and finding cloud-based services to be the most economical choice are the main factors that drive cloud migration. It was also highlighted by participants in Stage 1 that for an organisation which wishes to set-up services more quickly, the cloud can offer what they cannot do internally. A cloud provider stated:

\section{"One of the other things about the cloud that is attractive, but seems to come secondary to cost, but actually, is probably more important, is around agility and the ability to get new services much quicker than they can do internally."}

Enterprises can add or remove services as their businesses develop. In a technical sense, agile capabilities offered by the cloud enable enterprises to be much leaner. For example, a company in the UK decided to move to the cloud because they had a problem with agility, their internal processes and systems were too slow for what they wanted to do.
Cloud back-up was also highlighted by the participants as a perceived adavatage that can encourage organisations to migrate existing systems to the cloud. Cloud computing capabilities can enhance the availability of organisations systems by storing their data in a secure off-site backup. In the survey, a Technical Leader within a cloud provider company indicated that:

\section{"It doesn't really make any sense to have an on-site facility that need maintenance with hardware that may fail, while it can be deployed in the cloud at a very low cost, higher performance and easy disaster recovery."}

Cloud back-up can significantly reduce the risk of impact as the two versions would be running at the same time in different locations with the ability of moving data across the two environments.

The analysis of the information in this section led to the formulation of the following hypothesis:

H1: Organisations that perceive high relative advantages of cloud computing are more likely to migrate

Complexity The multi-user nature of cloud computing, as well as remote access, raises questions in respect of privacy and data confidentiality. Participants indicated that privacy and data confidentiality were their primary concerns in respect of the cloud environment. Further, interoperability was indicated by participants as the main perceived problem. Although cost reduction has been perceived as the main advatage that drives organisations to move to cloud computing, the analysis of stage 1 showed that the cost of cloud services emerged as an issue that some organisations faced when migrating to the cloud. Further, It was mentioned by the participants (cloud provider) that cost is the main reason for enterprises to back from the cloud. For example a cloud provider pointed out that "Costs are interesting because it's an instant attraction, but actually, very often clouds tends to be more expensive than people think it's going to be".

Cloud customers might need to pay for: data transfers in and out, storage of data in the cloud on a gigabyte per month basis, support, and additional availability. Therefore the combination of the charges a month actually become a considerable cost. A cloud provider pointed out that:

"I think one of the first things to remember with a cloud is that many clients look at cloud as being a cheap option because they see a few cents per hour price. But that few cents per hour is usually the base 
cost and then various additional services are added on, on top of it."

Further, cloud-based services are not always more economical than in-house provisions [45]. This depends upon the size of organisations and also on how long data is held. Usually, cloud computing is more cost effective for start-up companies or newly started organisations. On the other hand, companies that have large legacydata will find migration more difficult and costly. For these organisations, cloud services would be more beneficial for the provision of new services, but not to replace or migrate already deployed services. The Reasons that emerged for moving back to locally managed IT resources are: migration to the cloud without having full knowledge of the cost of cloud-services; the cost of support; consultancy and other services which were expected to be provided without additional cost; cloud services running beyond working time, Failure on ROI; vendors' standards.

Participants also pointed out that the provision of cloud-based services is different from the traditional offering. The differences include the pricing and payment methods, performance monitoring, regulation and compliance, architecture, security, and service support. These differences require a certain expertise that enterprises do not usually have.

One interviewee from a cloud engineering company pointed out:

"The reason that we exist, and there are a growing number of these businesses, is because customers do not have the expertise to migrate to the cloud."

It also emerged from the interviews that cloud customers have high expections of the level of support. Many of the cloud services are self-administered whereas many customers are expecting 24/7 support.

A security analyst interviewed indicated that "a lack of knowledge about the cloud environment is the riskest part of migrating to the cloud". It was suggested by a number of interviewees that there is a need to develop an understanding about general security of the cloud environment. This will aid organisations to define their requirements and ensure a clear division of responsibilities with regard to Service Level Agreement (SLAs). It was noted that enterprises need to continually review the cloud market, due its dynamic costing nature and the services offered, for example, the cost of bandwidth will change, as will the cost of services. Therefore, it can be concluded that cloud computing has created more challenges for IT managers, because in the cloud IT management roles need to be shifted into technology implementation. The translation requires practical skills and an understanding of how to securely implement cloud services.

H2: Perceiving cloud computing as a complex technology will negatively affect the decision to migrate

Trialability Testing in the cloud environment emerged from the survey as an important advantage. Enterprises can use the virtual hardware for a period of time and then turn it off, whereas many organisations simply cannot afford to have, for example, ten spare physical servers available to be used for testing for a short time, and then turn them off.

H3: Ease of testing in the cloud will positively influence the decision to migrate

Risks The process of migration to the cloud may involve: a number of risks, loss of privacy, disruption to business processes, legal implications, problems with interoperability, data integrity, application portability, and security issues [20, 46-48]. The Cloud Security Alliance [49] identified 7 top threats in respect of cloud computing. These threats had led to wide concerns about the availability and accessibility of cloud based services. The IT managers and security professionals interviewed in this study expressed their concerns over security with regards to the migration of sensitive data. Further, the analysis showed concerns about potential risks to organisations that may include: loss of control, dependability, managing relationships with different cloud providers, liability, and business continuity and disaster recovery. For example, if an organisation is unable to access cloud services at a critical time, when it is largely depending on the cloud provider's customer service to provide information, this can lead to poor customer satisfaction. Therefore, it can be concluded that the possibility or even the perception of high risks can negatively affect the decision to migrate.

H4: High perception of risks will negatively influence the decision to migrate

\section{Technology context}

Compatibility Lack of standards in cloud computing usually raise interoperability, mobility, and manageability issues between cloud providers. This may increase the likelihood of vendor lock-in with possible economic impacts [33].

In cases where organisations need to implement a service tailored to their needs, cloud computing may only provide part of the solution. In such a case, the enterprise may require additional technical expertise to 
integrate the cloud solution with the in-house system so-called 'hybrid cloud computing'.

Enterprises also need to evaluate the impact of migration to the cloud on the organisational culture and staff, due to possible unfamiliarity with the system and the environment. If a company wishes to migrate an existing system that has been developed and tailored over time with members of staff becoming accustomed to it, training would be required.

H5: The perception that cloud computing is less compatible with existing systems will negatively affect the decision to migrate

Size Organisations may need to predict their data volume as it has a direct impact on cost calculations. Usually, cloud computing is more cost effective for startups or newly started organisations [45]. On the other hand, companies that have large legacy-data will find migration more difficult and more expensive. For these organisations, cloud services, may be more beneficial for the provision of new services but not to replace, or migrate and deploy existing services.

H6: High volumes of data are less likely to be migrated

\section{Organisation context}

Readiness The heterogeneity of systems within organisations and their requirements have also affected the complexity of the decision making process when considering migration to cloud based solutions. Andrikopoulos et al. [6] pointed out that the decision whether to migrate, which cloud services to use and at which level to outsource is not trivial and that it largely depends on multiple factors that are specific to the context of each enterprise. Further, organisations have different business processes and interdependent criteria and constraints to consider when moving their systems to cloud environments [50]. The analysis of the Stage 1 also revealed that organisations have different expectations and understanding about the cloud environment.

The management related functions will become more important; for example, supplier relationships, service planning, contracts, negotiations, pricing, and procurement. These functions are required because enterprises need to maintain relationships with one or more cloud service providers that they have not used before. Further, organisations still need IT departments to monitor the cloud-based services and liaise with cloud providers for an effective system integration. Migration to the cloud will free IT managers from the burden of worrying about hardware, and they can focus on delivering better services. For example, one of the interviewees stated:
"So actually, it does change their perspective, but they actually focus on, 'what do my users need? What would be a better quality of service? What's a better performance time? And completely remove themselves from the hardware level."

The transformation of IT services has raised the need for new skills to deal with cloud-based services. It shifted roles and responsibilities from the local building and the support of internal resources to managing companies' systems in the cloud such as configuration, monitoring and integration of cloud services with the remaining on premise systems. These differences require a type of expertise that enterprises do not usually have.

H7: Organisations readiness will positively influence the decision to migrate to the cloud

\section{Internal social network}

The need for adapting existing services to be cloud enabled discussed in the interviews is a barrier against migrating existing systems to the cloud. According to [51] the systematic and efficient modernisation of legacy applications to exploit current cloud-based technologies remains a major challenge. Applications consist of several components that are connected with each other to comply with the application's functional and non-functional requirements [50]. These components need to be correctly adapted according to the target cloud environment. Failure or incorrect adaptation might result in difficulties in meeting some quality or economic requirements [50]. Typical adaptation problems range from compatibility and interoperability issues to licensing that may forbid organisations from moving registered software components.

Service performance may also be affected due to the increase in latency. These kinds of issues usually occur when a service component is shifted to the cloud while another dependent component is kept on-premises to meet security requirements [50]. Zhao and Zhou [52] identified the need for a holistic methodology from redesign and adaptation to application for special migration, architecture refactoring, integrated development environment and support migration of legacy applications to the cloud environment.

The security analysts interviewed in this study highlighted the importance of conducting risk analyses, because the business was originally based around a different type of architecture, and moving to the cloud could is seen as a dangerous way of doing business. The transfer may be risky and enterprises may need to adopt a different form of risk management going forward. However, if a business is newly-created, and it is based on the cloud and expands because of the cloud, then there should not be an issue. 
Migrating an existing system may result in errors and significant costs to the changes involved. Organisations do not want to see disruptions to their business and many enterprises are anxious that system failure may affect their reputations. From the security analyst's perspective, the security experts interviewed in this study indicated that it is a risky move when a company that revolves around its central service, needs to give up what the entire business is anchored upon and move to a cloud solution. However, companies may use the migration as an opportunity to improve their systems and processes.

H8: The impact of migration on the internal social network will negatively influence the decision for migration

\section{External social network}

The cloud providers interviewed in this study indicated that cloud capabilities allow organisations to accomplish their business operations more effectively by sharing information and work cooperatively. The cloud environment offers platforms and tools in which information can be easily accessed internally and externally, thus enhancing collaboration with internal co-workers and external stakeholders.

Ferrer et al. [53] stated that economic factors are not sufficient for a highly dynamic environment in which relationships are created on an on-off basis with a possible high degree of anonymity between stakeholders. A broader perspective is required that should incorporate quality factors such as trust, reputation management and green assessment.

H9: The impact of migration on the external social network will positively influence the decision for migration

\section{Top management support}

Top management support plays an important role for the decision to migrate to cloud computing. It guides the allocation of resources, the integration of services, and the re-engineering of processes. Top management that recognizes the benefits of cloud computing are likely to allocate the necessary resources for its adoption and influence the organization's members to implement the change. When top management fails to recognize the benefits of cloud computing to the business, the management will be opposed to its adoption [54].

An outsourcing culture is a main factor for migration to cloud computing. Trust is a major factor that supports the decision-making for cloud migration, and an outsourcing culture can develop trust of migration systems to a third party.
H10: Upper management support is positively related to the decision to migrate

\section{Environmental factors}

Expansion in the number of services, providers and configurations Another factor that influences the complexity of migration decisions is the availability of vast numbers of cloud-based services that have different models, functionality, quality of service, costs, and configurations. The range of cloud-based services offered is growing simultaneously with the emergence of varying cloud service providers. Enterprises can find cloud based models of possibly everything from general-purpose applications such as email, and collaboration technologies to sales management and accounting software [55]. This rapid increase opens up new opportunities for designing new applications and enterprise architectures, new quality levels and capabilities. It increases the difficulty of choosing a provider and a service and renders the run-time adaptation and replacement of services almost impossible [20]. According to García-Galán et al. [56] there are over 100 public cloud providers associated with a considerable number of configurations, for example Amazon web services has 16,991 different configurations.

H11: The process of selecting a cloud provider is difficult which negatively influences the decision to migrate

Regulation The increase in the number of rules to regulate the adoption of cloud services, regulations and legal compliance violation has become a concern for many organisations.

Extensive concerns over regulation and legal compliance violations were indicated by the participants in Stage 1. Participants also registered concerns about the loss or misuse of data by others, SLA problems, data ownership and intellectual property rights. It was argued that enterprises need to ensure compliance with regulations. For example, one of the interviewees stated that:

"We have to be slightly careful with this when we're accepting payments online, we just have to be careful not to fall foul of things like tax, VAT and so on."

A cloud provider indicated that they used to run systems for various organisations that held credit card information. They removed all the payment card information from their servers because they were affected by regulations on payments and currently they link their system to a payment card service provider. Further, the interviewees showed concerns about the location of 
datacentres. If a company migrates to a multinational service provider, it may find some of its resources stored in other parts of the world, a situation that can create legal issues.

The simplicity of signing up new cloud based service contracts might result in SLA problems. SLA is a vital aspect in cloud computing. It includes agreements regarding the quality of service attributes, pricing, compliance to regulation, level of support, security and privacy guarantees, and others. Organisations need to review the general terms and conditions that providers usually include in SLAs.

H12: Concerns about legal implication are negatively related to the decision to migrate

Difficulties in information gathering about the cloud market Generally, information plays an important role in any decision making process, particularly during the intelligence and design phases of a decision making process. Participants perceived that there is a high level of availability of cloud related information. Although many organisations believe that cloud-related information can be easily obtained and is to some extent it is reliable, they do not find it easy to develop an understanding about the cloud environment. It can be due to the diversity of information sources and their complexity. This has resulted in uncertainty about the cloud market. It could be the reason for the issue of limited knowledge of customers in respect of the cloud environment.

H13: Difficulties of information gathering will negatively influence the decision for migration

\section{Stage 2: testing the proposed model}

To evaluate the proposed model, a survey questionnaire was conducted using a wide range of industries and organisations' sizes. The questions were based on the findings of Stage 1 (see Table 2) as well as the analysis of related literature. To ensure that the survey measured what was intended, a pilot survey was first conducted. Four participants were used for the pilot study. The feedback showed that the questions were understandable and the participants were able to provide the required answers, though a few amendments were suggested.

Zikmund [57] suggested that the target population is the entire group of subjects of interest who are defined by the research objectives. However, there is usually a considerable difference between the population that a researcher is attempting to study and their availably for sampling [58]. The sample population in this study are targeted professionals with experience in related disciplines and users. The sample in this study is convenient sampling in which the researcher attempted, as far as possible, to find participants from the target audience by distributing the questionnaire using various methods. The target audience were mainly invited by e-mails through personal contacts. The questionnaire was distributed to more than 1,000 professionals and potential users and 118 responses were received. Sixteen responses were incomplete, therefore they were eliminated from the analysis leaving 102 usable responses which is approximately ten per cent of the total population and consistent with what could be expected for a survey of this kind.

The population consisted of professionals who had been involved in cloud migration projects or they were researchers in the area. Participants were from organisations of different sizes and from a divers industry sectors. They included IT managers, software engineers, system analysts, and executive managers; in addition to cloud systems researchers. The survey reflected the issues which had been raised in Stage 1 affecting migration to cloud computing. Figure 5 shows demographic data of participants and their organisations.

The data was imported from the survey tool (survey Monkey) into an IBM SPSS sheet. The reliability of the questionnaire was calculated which was followed by exploratory factor analysis and confirmatory factor analysis. The constructs (relative advantages, complexity, testing, risks, compatibility, size, readiness, impact on internal social network, external social network, top management support, increasing service providers and configuration, regulatory, and uncertainty about the market) were measured using a five-point Likert scale on an interval level ranging from 1 (very low importance) to 5 (very high importance). To investigate how the determinants vary across different industries, organisation sizes, and relation to the cloud, the data was analysed, based on these indicators. For example, lower scores indicate low influence of a variable on the decision to migrate. However, the results show no major differences between different industries, therefore this paper discusses the overall results only.

\section{Structural equation modelling}

To test the research hypotheses, Structural Equation Modelling (SEM) [59] was used. SEM is a statistical approach for exploring the relationships between observed variables and latent variables. It includes two main components: the measurement model and the structural model. The measurement model shows relationships between latent variables and observed variables. It aims to provide reliability and validity, based on these variables. The structural model measures path strength and the direction of the relationships among the variables. It is first necessary to test the measurement 


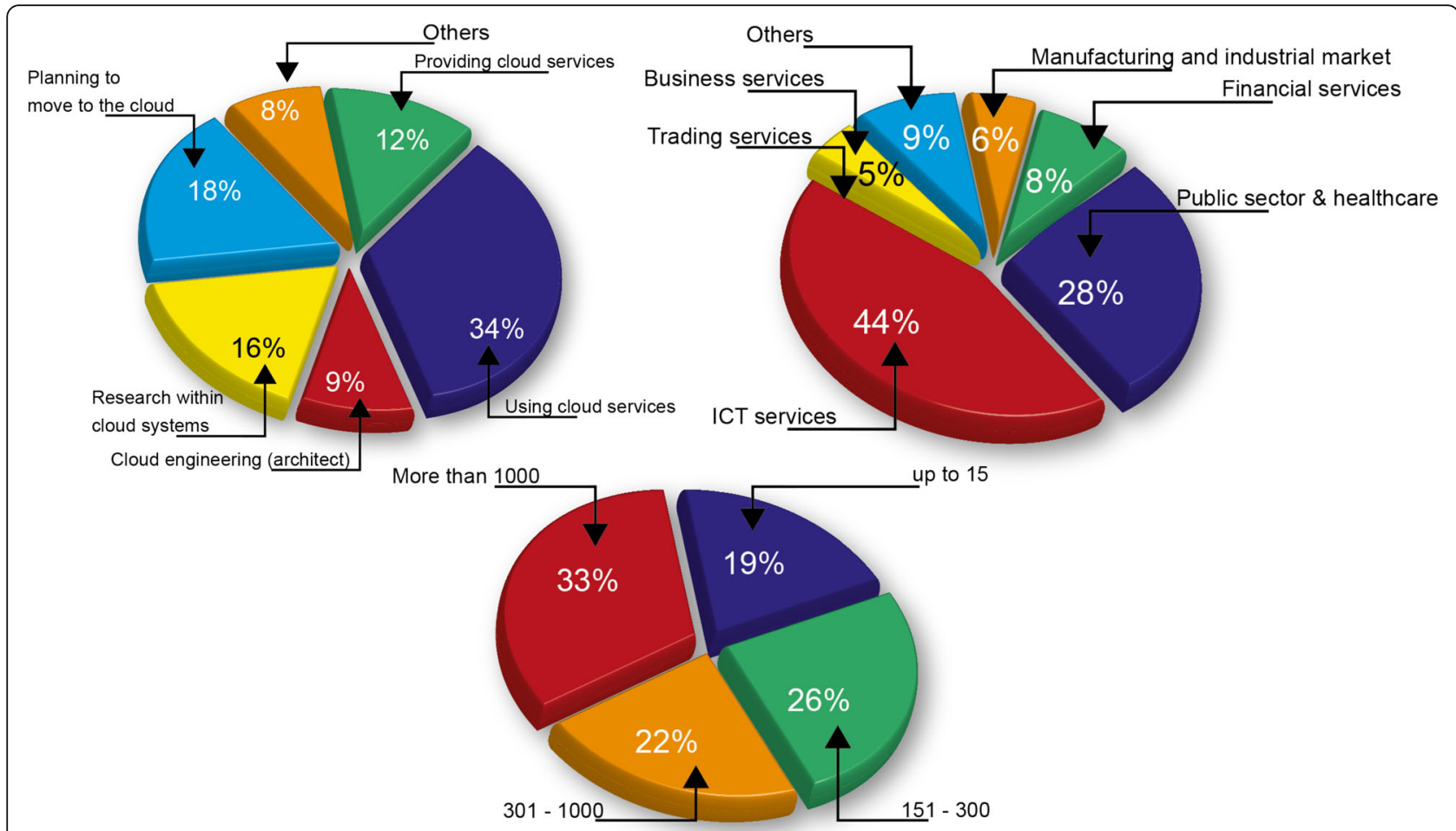

Fig. 5 Demographic of participants and their organisations

model and ensure that it has a satisfactory level of reliability and validity before exploring the significance of the relationships in the structural model.

\section{Exploratory factor analysis}

The result of applying the measurement model (reliability, convergent validity, discriminant validity, and descriptive statistics) are shown in Tables 4, 5, and 6. In order to test construct reliability for a set of two or more constructs (i.e. to examine internal consistency), the reliability of the scales was tested using composite reliability (CR) (more details may be found in [59]). The Cronbach's alpha [58] is a widely adopted method for testing $\mathrm{CR}$. It generates coefficient values ranging between 0

Table 4 Reliability of reflective constructs and $\mathrm{KMO}$ and Bartlett's Test

$$
\text { Reliability Statistics }
$$

Cronbach's Alpha

Results of the KMO and Bartlett's Test

Kaiser-Meyer-Olkin Measure of Sampling Adequacy

Approx. Chi-Square and 1 with higher values indicating higher reliability of the indicators. Fornell and Larcker [60] indicated that CR should have a value greater than 0.70 for a respectable research quality. The calculation formula for CR is: ( $\Sigma$ standardised loading $) 2 /\left(\sum\right.$ standardised loading $) 2+\Sigma \varepsilon$ ) where $\varepsilon=$ error variance and $\Sigma$ is summation.

Composite reliability analysis shows a Cronbach's " $\alpha$ " value of 0.773 for the 13 variables in the research model, which indicates high reliability and internal consistency (see Table 4). Further, the scale was factor analysed using principal component analysis.

In order to analyse the strength of association among the variables, the constructs validity was tested using the Bartlett's Test of Sphericity and the Kaiser-Mayer-Olkin (KMO) for the measure of sampling adequacy [61]. The result for Bartlett's test of Sphericity and the KMO value was 0.000 and 0.688 respectively (see Table 4 ). The latter value is more than 0.5 , which shows a high level of sampling adequacy.

Examining the correlation (Table 5) shows the highest level of correlation between constructs regulation and top management $(0.82)$. This is followed by correlation between compatibility and size of data $(0.72)$ as well as regulation and increasing providers $(0.77)$.

Discriminant validity refers to the extent to which a construct is truly distinct from other constructs [58]. A widely used statistical measure for discriminant validity 


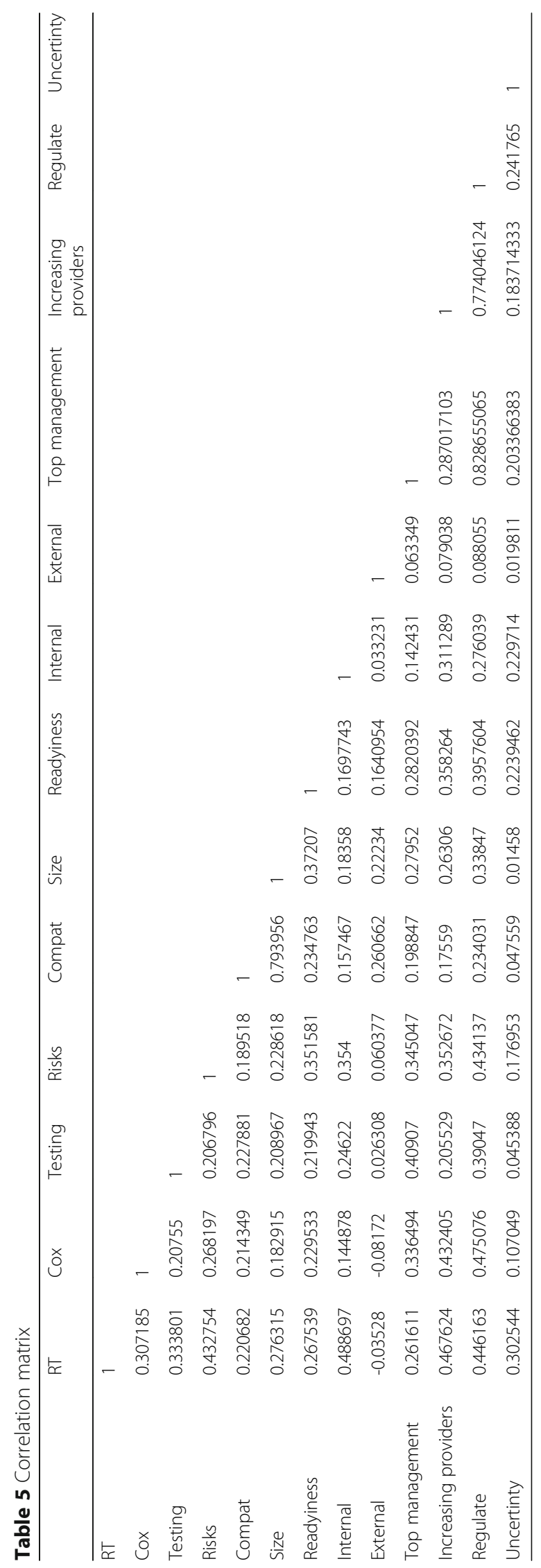


Table 6 Descriptive statistical

\begin{tabular}{|c|c|c|c|c|c|}
\hline Variable & Minimum & Maximum & Median & Mean & Standard Deviation \\
\hline 11 Cost Reduction & 3.0 & 5.0 & 4.0 & 4.45 & 0.71 \\
\hline I1Agility & 2.0 & 5.0 & 4.0 & 4.24 & 0.91 \\
\hline I1 Back-up & 2.0 & 5.0 & 4.0 & 4.09 & 0.80 \\
\hline 11 Higher performance & 2.0 & 5.0 & 4.0 & 4.20 & 0.68 \\
\hline I1 Average & 2.25 & 5.0 & 4.0 & 4.24 & 0.77 \\
\hline 12 Lack of knowledge & 2.0 & 5.0 & 4.0 & 4.18 & 0.83 \\
\hline 12 Cost management & 2.0 & 5.0 & 4.0 & 3.54 & 1.04 \\
\hline 12 Risk management & 3.0 & 5.0 & 5.0 & 4.66 & 0.58 \\
\hline 12 Average & 2.0 & 5.0 & 4.0 & 4.12 & 0.86 \\
\hline 13 Testing & 3.0 & 5.0 & 4.0 & 3.98 & 0.80 \\
\hline 14 Privacy and confidentiality & 3.0 & 5.0 & 5.0 & 4.39 & 0.75 \\
\hline 14 Vendor lock in & 1.0 & 5.0 & 4.0 & 3.72 & 1.03 \\
\hline 14 Loss of control & 2.0 & 5.0 & 4.0 & 4.26 & 0.79 \\
\hline 14 Average & 2.0 & 5.0 & 4.3 & 4.12 & 0.89 \\
\hline T1 Impact on culture and staff & 1.0 & 5.0 & 5.0 & 3.49 & 0.94 \\
\hline T1 Interpretability issues & 2.0 & 5.0 & 4.0 & 4.22 & 0.83 \\
\hline T1 Average & 1.5 & 5.0 & 4.5 & 3.85 & 0.88 \\
\hline T2 Size & 1.0 & 5.0 & 4.0 & 3.56 & 0.99 \\
\hline O1 Current IT infrastructure & 1.0 & 5.0 & 4.0 & 4.15 & 0.77 \\
\hline O1 Level of expertise & 1.0 & 5.0 & 3.0 & 3.71 & 0.90 \\
\hline O1 Average & 1.0 & 5.0 & 3.5 & 3.92 & 0.99 \\
\hline O2 The need for adaptation & 1.0 & 5.0 & 4.0 & 3.88 & 1.03 \\
\hline O2 Disruption to BP & 1.0 & 5.0 & 4.0 & 3.84 & 0.91 \\
\hline O2 Average & 1.0 & 5.0 & 4.0 & 3.86 & 0.97 \\
\hline O3 Collaboration & 1.0 & 5.0 & 4.0 & 2.84 & 1.17 \\
\hline O4 Top management support & 1.0 & 5.0 & 4.0 & 4.15 & 0.77 \\
\hline E1 Provider selection & 1.0 & 5.0 & 4.0 & 4.24 & 0.84 \\
\hline E1 Increasing providers and configurations & 1.0 & 5.0 & 4.0 & 4.16 & 0.78 \\
\hline E1 Average & 1.0 & 5.0 & 4.0 & 4.20 & 0.81 \\
\hline E2 Legal implication & 1.0 & 5.0 & 4.0 & 4.22 & 0.76 \\
\hline E2 IP & 2.0 & 5.0 & 4.0 & 4.32 & 0.71 \\
\hline E2 SLA & 2.0 & 5.0 & 4.0 & 4.20 & 0.81 \\
\hline E2 Average & 1.6 & 5.0 & 4.0 & 4.25 & 0.76 \\
\hline E3 Accessibility & 2.0 & 5.0 & 4.0 & 3.81 & 0.88 \\
\hline E3 Complexity & 2.0 & 5.0 & 3.0 & 3.0 & 0.89 \\
\hline E3 Average & 2.0 & 5.0 & 3.5 & 3.49 & 0.89 \\
\hline
\end{tabular}

is a comparison of the Average Variance Extracted (AVE) with correlation squared [62]. In order to ensure discriminant validity, the AVE of two constructs must be more than the square of the correlation between the same two constructs. Our results show that AVE mean square root of each value variable is significantly greater than its correlation coefficient with other variables (see Table 5), thus discriminant validity is supported. Overall, the results show acceptable reliability, convergent validity and discriminant validity, which were appropriate for testing the research model.

Table 5 provides the correlation matrix for discriminant validity, which indicates the degree to which the constructs diverge from each other.

\section{Confirmatory factor analysis}

To test the stability of the scale, confirmatory factor analysis was employed using SEM to examine the 
hypothesized research model by performing a simultaneous test. A measurement model was developed using the AMOS tool and Maximum Likelihood Estimation (MLE) method was chosen for confirmatory factor analysis. MLE is a common estimation procedure used in SEM software. It does not require large sample size and the results of the method are reliable. Therefore, it is widely used in theoretical hypothesis testing. The structural model shows path coefficients results. It illustrates the extent of the mutual influence among variables. Path coefficient was calculated automatically by the tool. Figure 6 shows the standardized results for the structural model.

\section{Results and discussion}

The results of the analysis of Stage 2 reveal that 10 out of the 13 variables identified in the research model (see Fig. 4) significantly influenced the decision making for migrating to the cloud. Size of data volume, testing, and the impact on external network are not significant factors.

The Relative advantages factor is supported as a positive influence on the decision to migrate existing resources to the cloud. It has a significant $(p<0.05)$ and positive coefficient of 0.18 , when considering migrating to the cloud. Within this variable the cost benefits factor was rated the highest (4.45) followed by agility (4.24), higher performance (4.20), and the back-up advantages (4.09) as shown in Table 6. This finding confirms the results found in similar previous studies that identified perceived advantages as the most influential factor for adopting cloud services (see for example: [9, 37, 38, 62, 63]). The realisation of the relative advantages shows that organisations perceive the cloud environment as a platform for rationalising expenditure and improving efficiency. Organisations need to review the cloud offers and appreciate how the advantages of the cloud provisions can be exploited in improve their business performances. This can lead to improvements in customer service, cost management, relationship with customers and stakeholders, management structure and policies, and business objectives.

The complexity of the cloud environment factor is confirmed in Stage 2 as a negative influence for the decision to migrate to the cloud, with path coefficient of $(-0.14)$. Within this variable organisations, perceived conducting a risk management for the migration as very difficult with an average rating of 4.47 . This is followed by lack of

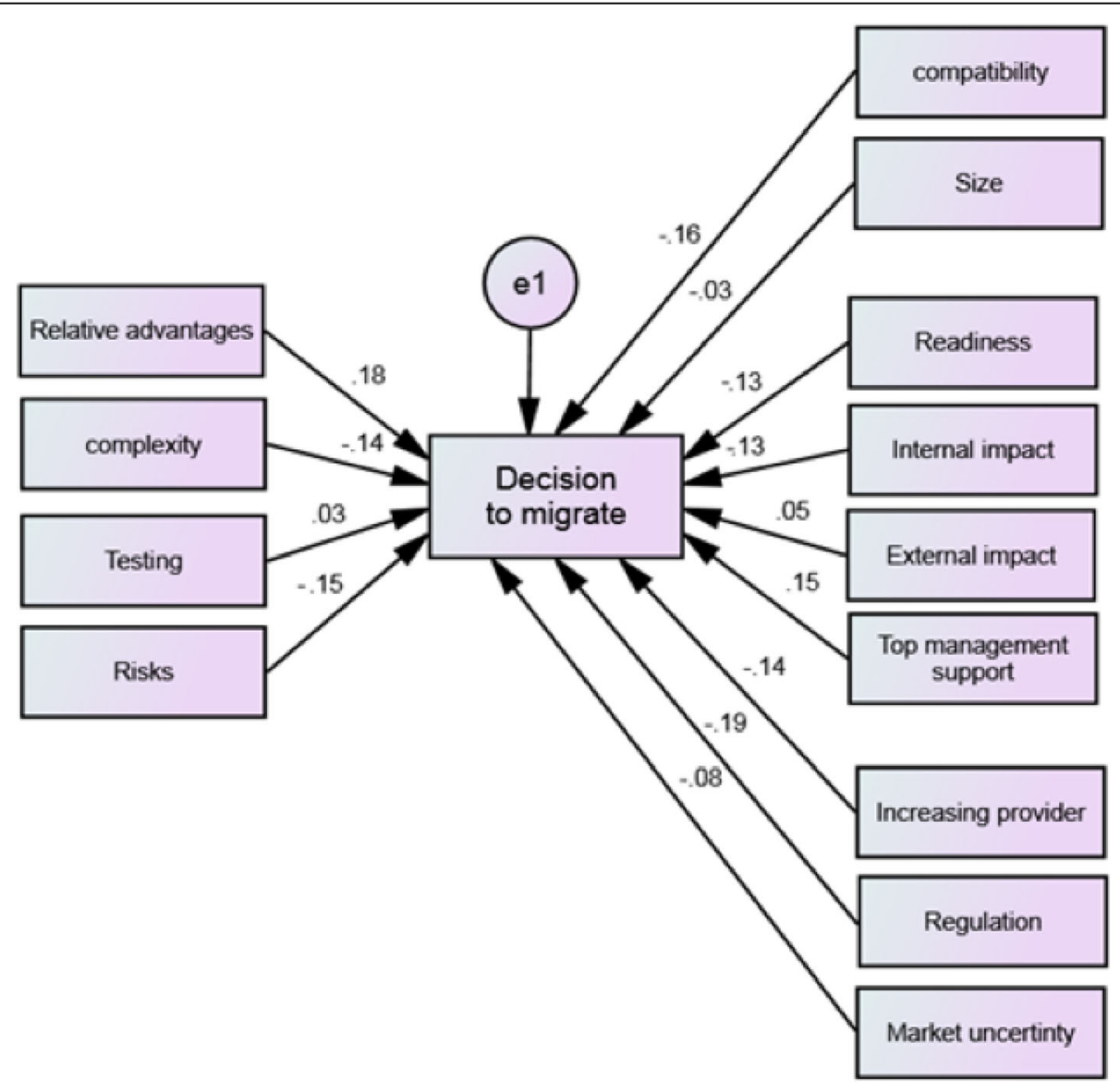

Fig. 6 Structural equation modelling using AMOS tool. e1: error variance 
knowledge about the cloud environment with an average rating of 4.03 and the complexity of managing costs (3.62). Pervious works showed different results regarding the perceived complexity of cloud environment. Oliveira et al. [63] presented results in line with these findings; however, other studies suggested the opposite that the cloud environment is not perceived as a complex technology [13] and [64]. Cloud computing is a disruptive model of provisioning IT services, therefore perceiving it as complex environment is expected. The complexity can be mitigated by taking advantage of the testing facilities in the cloud that can allow organisations to be more familiar with the new environment. Further, the cloud is still in a growing phase and its complexity and risks are expected to be reduced.

Although ease of testing in the cloud environment is perceived by many as an advantage encouraging organisations to migrate to the cloud, it is not supported by our findings as a critical influencing factor for the decision to migrate. This may be due to the fact that the majority of the organisations who participated in this study are small and medium size (SME) and testing is not a critical factor for them. Further, testing would be more relevant to larger organisations such as universities and research centres which are not highly represented in this study.

The perception that migrating to the cloud is a risky decision factor is found to negatively affect companies' decisions to migrate to the cloud. This factor scored a path coefficient of -0.15 . Within this category privacy and confidentiality scored the highest with an average rate of 4.21 followed by the concerns of losing control rated at 4.09, and the concerns of vendor lock-in scored an average rating of 3.73 . This result substantiates similar studies that conducted at the beginning of cloud adoption such as [13-15]. Therefore, security, fear of losing control, and vendor lock-in are still perceived by many as issues deterring migration.

Within the technology context, the compatibility factor is confirmed to as a negatively influence on the decision to migrate with a path coefficient of -0.16 while the size of data volume factor is not supported as an important factor for the decision to migrate with a path coefficient of -0.03 . Within this variable, impact on culture and staff scored an average rating of 3.49 and interpretability issues obtained 4.22 . To manage the impact on staffing, organisations need to analyse the anticipated change to the staffing level, roles and expertise during the design phase, before commencing the process of migrating existing services to the cloud.

In respect of the organisational context, readiness, the impact on internal networks, and top management realisation of the business benefits factors have significant effects, with path coefficients of $-0.13,-0.13,0.15$ respectively, while the impact of external network factor does not (0.05). This result shows that organisations that have higher levels of readiness require less adaptation, support from top management for cloud environment are more likely to migrate.

In the environment context, all variables have a significant effect, in particular the regulation factor, with a path coefficient of - 0.19 which is the highest influencing factor. The increasing service providers factor scored path coefficient of - 0.14 and the uncertainty about the market factor obtained - 0.08. Many organisations comply with regulators in their internal systems management, but by adopting cloud services, part of their service management can be shifted to the cloud service provider. In this scenario organisations need to know how to continue their compliance with the regulators which could be a challenge. Organisations need to review the general terms and conditions that providers usually include in SLAs. Companies need to review vendors' standard contracts, to see if their basic terms are sufficient for their organisational compliance requirements, and to ensure service providers' compliance with their regulators. Table 7 shows the results of testing the research hypotheses.

In order to improve trust in the cloud services, providers need to ensure privacy and data confidentiality of their customers. Further, complying with relevant standards would help in reducing the risks of vendor lock-in. This issue is partly caused by the lack of standardisation in cloud computing which was also indicated by participants as an issue that contributed to the complexity of cloud computing. In order to help organisations to decide whether to migrate to the cloud, there is a need to inform them about general security; whether the security of the infrastructure is resilient. The project manager should then move to the performance aspects by providing information about the opportunities and options which will meet the customer's requirements. The decision makers may not be interested in the technical details but it is important for them to see the business benefit. These items of information will allow enterprises to build confidence in the cloud service management. Based on this information, it should be possible to advise enterprises which cloud solution will be most suitable for them. Therefore, providers need to ensure the privacy and confidentiality of their customers' data, employ comprehensive costing models, in addition to complying with relevant standards to reduce the risks of vendor lock-in. These could remedy the high level of perceiving the migration to the cloud as a risky decision.

\section{Contribution}

This paper focused on the decision to migrate existing resources to the cloud. It empirically explored factors 
Table 7 Results of hypotheses testing

\begin{tabular}{|c|c|c|c|}
\hline No & Hypotheses & Coefficient & Result \\
\hline $\mathrm{H} 1(+)$ & $\begin{array}{l}\text { Organisations that perceive high relative advantages of cloud computing } \\
\text { are more likely to migrate }\end{array}$ & 0.18 & Supported $(p<0.05)$ \\
\hline $\mathrm{H} 2(-)$ & $\begin{array}{l}\text { Perceiving cloud computing as a complex technology will negatively } \\
\text { affect the decision to migrate }\end{array}$ & -0.14 & Supported $(p<0.05)$ \\
\hline $\mathrm{H} 3(+)$ & Ease of testing in the cloud will positively influence the decision to migrate & 0.03 & Not supported \\
\hline $\mathrm{H} 4(-)$ & High perception of risks will negatively influence the decision to migrate & -0.14 & Supported $(p<0.05)$ \\
\hline H5 (-) & $\begin{array}{l}\text { The perception that cloud computing is less compatible with existing } \\
\text { systems will negatively affect the decision to migrate }\end{array}$ & -0.16 & Supported $(p<0.05)$ \\
\hline H6 (-) & High volumes of data are less likely to be migrated & -0.03 & Not Supported \\
\hline $\mathrm{H} 7(+)$ & $\begin{array}{l}\text { Organisations readiness will positively influence the decision to migrate } \\
\text { to the cloud }\end{array}$ & -0.13 & Supported $(p<0.05)$ \\
\hline H8 (-) & $\begin{array}{l}\text { The impact of migration on the internal social network will negatively } \\
\text { influence the decision to migrate }\end{array}$ & -0.13 & Supported $(p<0.05)$ \\
\hline $\mathrm{H} 9(+)$ & $\begin{array}{l}\text { The impact of migration on external social network will positively influence } \\
\text { the decision to migrate }\end{array}$ & 0.05 & Not Supported \\
\hline $\mathrm{H} 10(+)$ & Upper management support is positively related to the decision to migrate & 0.15 & Supported $(p<0.05)$ \\
\hline H11 (-) & $\begin{array}{l}\text { The process of selecting a cloud provider is difficult which negatively } \\
\text { influences the decision to migrate }\end{array}$ & -0.14 & Supported $(p<0.05)$ \\
\hline $\mathrm{H} 12(-)$ & $\begin{array}{l}\text { Concerns about legal implication are negatively related to the decision } \\
\text { to migrate }\end{array}$ & -0.19 & Supported $(p<0.05)$ \\
\hline $\mathrm{H} 13(-)$ & $\begin{array}{l}\text { Difficulties of information gathering will negatively influence the decision } \\
\text { to migrate }\end{array}$ & -0.8 & Supported $(p<0.05)$ \\
\hline
\end{tabular}

influencing the decision to migrate by designing a model, based on TOE and DOI frameworks. The findings of this research has expanded the collective knowledge about the complexity of the issues that have to be considered when making decisions to migrate to the cloud. Seven determinants were identified that influence the complexity of decisions to migrate (see $\mathrm{H} 2, \mathrm{H} 4, \mathrm{H} 5$, H8, H11, H12, and H13 in Table 7). Some of the factors are similar to those which have been identified in previous studies that were conducted at an early stage of the evolution of cloud computing. This paper confirms that those factors are still being perceived as deterrent for migration. They are: H4, H5, and $\mathrm{H} 12$ while the other four are novel contributions unique to this paper. Further, the findings in this research are quite different compared to similar studies in that the majority of them explored the factors affecting the adoption of cloud services while this research focused on the migration of legacy systems.

The analysis in this paper shows that cloud computing is perceived by many as a valuable opportunity for business benefits such as cost reduction, agility, back-ups, and higher business performance. However, it identified a number of factors that increased the complexity of decisions to migrate. It highlighted how enterprises perceived the level of complexity of the cloud environment and the expertise required to design and manage cloud services. In particular, this research highlighted the lack of knowledge about the cloud environment. It also identified lack of expertise within organisations for design and management of cloud services. Further, our results confirm that cloud computing is in its early stages, and is still evolving. It also showed that financial benefits of cloud services vary and cost is found to be the main reason for enterprises that moved back from the cloud. Further, this study supported the view that since the emergence of cloud computing, security has been the main problem perceived by many organisations.

This study provides a better understanding of a wide range of factors that affect the decision making process for migration to the cloud. They can asset cloud DSS developers in the designing of systems that address their issue and concerns. Specifically, the application of the Simon's model for a systematic decision process for the cloud DSS shows that there is limited support at the intelligence and design phases. The analysis of this research shows a number of factors that require cloud DSS to provide support at intelligence (e.g. H2 in Table 7) and design (e.g. H5 in Table 7) levels. Therefore, this study contributes to the literature that addresses the complexity and multidimensional nature of migrating IT resources to the cloud.

\section{Conclusion}

Cloud computing is a new paradigm for emerging technologies. Migration to cloud computing is a strategic 
organisational decision that is complicated, dynamic, and highly unstructured. The cloud environment is still evolving; therefore, decision makers need to carefully evaluate the capabilities of cloud computing services to determine whether this style of computing will help them achieve their business goals. It requires the consideration and evaluation of a wide range of technical and organisational aspects. This research is aimed to support the decision making process for migrating existing resources to the cloud. It explored factors contributing to the complexity of the decision to migrate.

The review of current approaches for migration to the cloud revealed that, although many methods have been proposed to aid migration to it, the level of support offered by the existing DSS is not sufficient to enable decision makers to make informed decisions. Accordingly, many organisations are still finding it difficult to make decisions migrating existing resources to the cloud. This is mainly due to many complex factors that affect decision making to migrate to the cloud.

Understanding the level of complexity for making decisions to migrate aids the development of suitable resources and processes to simplify it. Accordingly, this paper extensively explored the factors affecting decision making. By integrating the innovation characteristics of the DOI and the TOE frameworks a model was developed. The model was empirically evaluated using the exploratory and confirmatory analysis, which identified the factors that have a significant positive or negative effect on the decision to migrate. A future work to enhance the findings of this study can be further examination of correlation between the identified variables. In addition, significant factors affecting migrations can be included in cloud DSSs.

\section{Acknowledgements}

The research leading to these results is supported by the faculty of science and Technology at Bournemouth University.

\section{Authors' contributions \\ All listed authors worked closely throughout the development of this research and made major contributions. AA was responsible for literature review and data collection and analysis. Dr RS and Dr DJ contributed in the designing of the methodology including the design of the research instruments. All authors participated in drafting the manuscript, analysis and interpretation of study results. RS and DJ participated in the critical and technical revisions of the paper including editing the final version. They supervised the project related to the paper and also gave final approval of the version to be published. All authors read and approved final manuscript.}

\section{Authors' information}

Adel Alkhalil is a recently PhD graduate from Bournemouth University, UK under supervision of Dr Reza Sahandi and Dr David John. In September 2016, Dr Alkhalil joined the faculty of computer science and engineering at the University of Hail, Saudi Arabia. His research interests include cloud computing, decision support systems, knowledge-based systems. Reza Sahandi completed his PhD at Bradford University in the United Kingdom in 1978. He has been a senior academic at various Universities in the United Kingdom for many years. He is currently Associate Professor at Bournemouth University. He has supervised many PhD students. His research areas include multimedia and network systems, wireless remote patient monitoring and cloud computing.

David John completed his PhD at in the United Kingdom in 2002. Since then he has been an academic member in the faculty of computer science and engineering at Bournemouth University. His research interests include cloud computing, expressive Internet communication, assessing the effect cognitive style has on the perception of different modes of communication, and the evaluation of creative practice-based assessment methods.

\section{Competing interests}

The authors declare that they have no competing interests.

\section{Author details}

${ }^{1}$ Faculty of Computer Science and Engineering, University of Hail, Hail, Saudi Arabia. ${ }^{2}$ Faculty of Science and Technology, Bournemouth University, Poole, UK.

Received: 24 May 2016 Accepted: 20 December 2016

Published online: 13 January 2017

\section{References}

1. Jennings B, Stadler R (2015) Resource management in clouds: Survey and research challenges. J Netw Syst Manag 23(3):567-619

2. Dhinesh Babu LD, Gunasekaran A, Krishna PV (2014) A decision-based pre-emptive fair scheduling strategy to process cloud computing work-flows for sustainable enterprise management. Int J Bus Inf Syst 16(4):409-430

3. Armbrust M, Fox A, Griffith R, Joseph AD, Katz R, Konwinski A, Zaharia M (2010) A view of cloud computing. Communications of the ACM 53(4):50-58

4. Buyya R, Yeo CS, Venugopal S, Broberg J, Brandic I (2009) Cloud computing and emerging IT platforms: Vision, hype, and reality for delivering computing as the 5th utility. Futur Gener Comput Syst 25(6):599-616

5. Phaphoom N, Wang X, Samuel S, Helmer S, Abrahamsson P (2015) A survey study on major technical barriers affecting the decision to adopt cloud services. J Syst Softw 103:167-181

6. Andrikopoulos V, Binz T, Leymann F, Strauch S (2013) How to adapt applications for the Cloud environment. Computing 95(6):493-535

7. Rai R, Sahoo G, Mehfuz S (2013) Securing software as a service model of cloud computing: Issues and solutions. International Journal on Cloud Computing: Services and Architecture (IJCCSA) 3(4):1-11

8. Oredo JO, Njihia JM (2015) Mindfulness and quality of innovation in cloud computing adoption, Int J Bus Manage, 10(1):144

9. Hsu PF, Ray S, Li-Hsieh YY (2014) Examining cloud computing adoption intention, pricing mechanism, and deployment model. Int J Inf Manag 34(4): 474-488

10. Buyya R (2009) Market-oriented cloud computing: Vision, hype, and reality of delivering computing as the 5th utility. In: 9Th IEEE/ACM International Symposium on Cluster Computing and the Grid, CCGRID

11. Mell P, Grance T (2009) The NIST Definition of Cloud Computing. Natl Inst Stand Technol 53(6):50

12. Leimeister S, Christoph R, Markus B, Helmut K (2010) The Business Perspective of Cloud Computing: Actors, Roles and Value Networks, European Conference on Information Systems

13. Low C, Chen Y, Wu M (2011) Understanding the determinants of cloud computing adoption. Ind Manag Data Syst 111(7):1006-1023

14. Wu WW (2011) Mining significant factors affecting the adoption of SaaS using the rough set approach. J Syst Softw 84(3):435-441

15. Sahandi R, Alkhalil A, Opara-Martins J (2012) SMEs' Perception of Cloud Computing: Potential and Security. In: Collaborative Networks in the Internet of Services. PRO-VE 2012. IFIP Advances in Information and Communication Technology, 380, Springer, Berlin, pp 186-195

16. Lin A, Chen N (2012) Cloud computing as an innovation: Perception, attitude, and adoption. Int J Inf Manag 32(6):533-540, Available from: http://www. sciencedirect.com/science/article/pii/S0268401212000539. Accessed 16 May 2016

17. Morgan L, Conboy K (2013) Factors affecting the adoption of cloud computing: an exploratory study., pp 1-12

18. Khajeh-Hosseini A, Greenwood D, Smith J, Sommerville I (2012) The Cloud Adoption Toolkit: Supporting cloud adoption decisions in the enterprise. Software - Practice And Experience 42(4):447-465

19. Menzel M, Ranjan R, Wang L, Khan S, Chen J (2014) Cloudgenius: A hybrid decision support method for automating the migration of web application clusters to public clouds. IEEE transactions on 64(5):1336-1348 
20. Omerovic A, Muntes-Mulero V, Matthews P, Gunka A (2013) Towards a Method for Decision Support in Multi-cloud Environments. In: the fourth International Conference on Cloud Computing, GRIDs, and Virtualisation., pp 244-250

21. Andrikopoulos V, Song Z, Leymann F (2013) Supporting the Migration of Applications to the Cloud through a Decision Support System. In: IEEE Sixth International Conference on Cloud Computing., pp 565-572

22. Garg SK, Versteeg S, Buyya R (2013) A framework for ranking of cloud computing services. Futur Gener Comput Syst 626(4):1012-1023

23. Saez S G, Andrikopoulos V,Hahn M, Karastoyanova D, Leymann F, Skouradaki M, Vukojevic-Haupt K (2015) Performance and Cost Evaluation for the Migration of a Scientific Workflow Infrastructure to the Cloud, 5th International Conference of Cloud Computing and Service Science, CLOSER 2015, Lisbon, Portugal, pp 1-10

24. Gonidis F, Paraskakis I, Simons AJ (2014) Leveraging Platform Basic Services in Cloud Application Platforms for the Development of Cloud Applications. In: IEEE 6th International Conference on Cloud Computing Technology and Science., pp 751-754

25. Pahl C, Xiong H, Walshe R (2013) A comparison of on-premise to cloud migration approaches. In: European Conference on Service-Oriented and Cloud Computing ESOCC., pp 11-13

26. Simon HA (1977) The new science of management decision, 2nd edn. Prentice Hall, Englewood Cliffs (NJ)

27. Liu S, Duffy A, Whitfield R, Boyle IND (2010) Integration of decision support systems to improve decision support performance. Knowl And Inf Syst 22(3):261-286

28. Turban E, Aronson J, Liang TP (2005) Decision Support Systems and Intelligent Systems, 7th edn., Pearson Prentice Hall

29. Oracle (2012) Cloud Integration - A Comprehensive Solution. Oracle

30. Amazon EC2 (2015). Available from: https://d0.awsstatic.com/whitepapers/RDS/ AWS_Database_Migration_Service_Best_Practices.pdf. Accessed 16 May 2016.

31. Accenture (2013) Putting the cloud to work with Accenture. Available from: https://www.accenture.com/t20150623T023251_w__/us-en/_acnmedia/ Accenture/Conversion-Assets/DotCom/Documents/Global/PDF/Technology_ 2/Accenture-Putting-Cloud-Work-Acn.pdf. Accessed 16 May 2016.

32. Khajeh-Hosseini A, Sommerville I, Bogaerts J, Teregowda P (2011) Decision support tools for cloud migration in the enterprise. In: Proceedings - 2011 IEEE 4th International Conference on Cloud Computing, CLOUD., pp 541-548

33. Opara-Martin J, Sahandi R, Tian F (2016) Critical Analysis of Vendor Lock-in and its Impact on Cloud Computing Migration: A Business Perspective. Journal of Cloud Computing Advances, Systems and Applications 5:4, Available from: http://link. springer.com/article/10.1186/s13677-016-0054-z. Accessed 16 May 2016

34. Tornatzky LG, Fleischer M (1990) The processes of technological innovation. Lexington Books, Lexington, MA

35. Rogers EM (2003) Diffusion of innovations, 5th edn. Free Press, New York

36. Ramdani B, Chevers DA, Williams D (2013) SMEs' adoption of enterprise applications: A technology-organisation-environment model. Journal of Small Business and Enterprise Development 20(4):735-753

37. Gangwar H, Date H, Ramaswamy R (2015) Understanding determinants of cloud computing adoption using an integrated TAM-TOE model. J Enterp Inf Manag 28(1):107-130

38. Lian JW, Yen DC, Wang YT (2014) An exploratory study to understand the critical factors affecting the decision to adopt cloud computing in Taiwan hospital. Int J Inf Manag 34(1):28-36

39. Tashkandi AA, Al-Jabri, Ibrahim M (2015) Cloud Computing Adoption by Higher Education Institutions in Saudi Arabia International Conference on Cloud Computing. Riyadh, Saudi Arabia, Available from: http://ssrn.com/ abstract=2624564. Accessed 16 May 2016

40. Prescott MB (1995) Diffusion of innovation theory: Borrowings, extensions and modifications from IT researchers. Data Base 26(2\&3):16-19

41. Chau PYK, Tam KY (1997) Factors affecting the adoption of open systems: an exploratory study. MIS Q 21:1-24

42. Alkhalil A, Sahandi R, John D (2013) Migration to Cloud Computing-The Impact on IT Management and Security. In: 1st International Workshop on Cloud Computing and Information Security. Atlantis Press, Shanghai, China, pp 196-200

43. Alkhalil A, Sahandi R, John D (2014) Migration to Cloud Computing: A Decision Process Model. In th 25th Central European Conference on Information and Intelligent Systems, CECIIS-2014. Faculty of Organization and Informatics Varazdin, Varazdin. pp 17-19

44. Fichman RG (2004) Going beyond the dominant paradigm for information technology innovation research: emerging concepts and methods. J Assoc Inf Syst 5:314-355

45. Misra S, Mondal A (2011) Identification of a company's suitability for the adoption of cloud computing and modelling its corresponding Return on Investment. Mathematical And Computer Modelling 53(3):504-521
46. Motahari-Nezhad H R, Stephenson, B, Singhal S (2009) Outsourcing business to cloud computing services: Opportunities and challenges. IEEE Internet Computing, 10(4):1-17

47. Sahandi R, Alkhalil A, Opara-Martins J (2013) Cloud Computing from SMEs perspective: a Survey based Investigation. J Inf Technol Manag 24(1):1-12

48. Willcocks L, Venters W, Whitley E A (2011) Clear view of the cloud: The business impact of cloud computing. Available from: https:/www.scribd.com/ document/65119786/Accenture-Outlook-Clear-View-of-the-Cloud-ComputingThe-Business-Impact-of-Cloud-Computing. Accessed 30 Dec 2016

49. CSA (2013) Practices for Secure Development of Cloud. Cloud security Alliance, Available from: http://www.safecode.org/publications/SAFECode_ CSA_Cloud_Final1213.pdf. Accessed 10 July 2013

50. Juan-Verdejo A, Baars H, Kemper H, Surajbali B, Zschaler S (2014) InCLOUDer: A formalised decision support modelling approach to migrate applications to cloud environments. In: Proceedings - 40Th Euromicro Conference Series On Software Engineering And Advanced Applications, SEAA., pp 67-474

51. Bergmayr A, Bruneliere $H$, Canovas Izquierdo JL, Gorronogoitia J, Kousiouris G, Kyriazis D, Wimmer M (2013) Migrating legacy software to the cloud with ARTIST. In: IEEE Software Maintenance and Reengineering (CSMR), European Conference., pp 465-468

52. Zhao JF, Zhou JT (2014) Strategies and methods for cloud migration. Int J Autom Comput 11(2):143-152

53. Ferrer A, Hernández F, Tordsson J, Elmroth E, Ali-Eldin A, Zsigri C, Sirvent R, Guitart J, Badia R, Djemame K, Ziegler W, Dimitrakos T, Nair S, Kousiouris G, Konstanteli K, Varvarigou T, Hudzia B, Kipp A, Wesner S, Corrales M, Forgó N, Sharif T, Sheridan C (2012) OPTIMIS: A holistic approach to cloud service provisioning. Futur Gener Comput Syst 28:66-77

54. Amini M, Bakri A, Sadat Safavi N, Javadinia SA, Tolooei A (2014) The Role of Top Manager Behaviours on Adoption of Cloud Computing for Small and Medium Enterprises. Aust J Basic Appl Sci 8(1):490-498

55. Gogrid (2012) Cloud computing adoption slower than expected, Available from: http://www.gogrid.com/news/2012 February 22/cloud-computingadoption-slower-thanexpected. Accessed 10 July 2013.

56. García-Galán J, Trinidad P, Rana O F, Ruiz-Cortés A (2016) Automated configuration support for infrastructure migration to the cloud, Futur Gener Comput Syst, 55, pp.200-212 Available from:http://www.sciencedirect.com/ science/article/pii/S0167739X15000618. Accessed 1 June 2015.

57. Zikmund WG (2000) Business Research Methods, 6th edn. the Dryden Press, Chicago, IL

58. Hair JF, Anderson RE, Tatham RL, Black WC (1992) Multivariate Data Analysis, 3rd edn. Macmillan, New York

59. Hoyle RH (1995) The structural equation modeling approach: Basic concepts and fundamental issues. In: Hoyle RH (ed) Structural equation modeling: Concepts, issues, and applications. Sage Publications, Inc., Thousand Oaks, CA, pp 1-15

60. Fornell C, Larcker DF (1981) Evaluating structural equation models with unob-servable variables and measurement error. J Market Res 18(1):39-50

61. Beavers AS, Lounsbury JW, Richards JK, Huck SW, Skolits GJ, Esquivel SL (2013) Practical considerations for using exploratory factor analysis in educational research. Practical assessment, research \& evaluation 18(6):1-13

62. Alshamaila Y, Papagiannidis S, Li F (2013) Cloud computing adoption by SMEs in the north east of England: A multi-perspective framework. J Enterp Inf Manag 26(3):250-275

63. Oliveira T, Thomas M, Espadanal M (2014) Assessing the determinants of cloud computing adoption: An analysis of the manufacturing and services sectors. Inf Manag 51(5):497-501, Available from: http://www.sciencedirect. com/science/article/pii/S0378720614000391. Accessed 16 May 2016

64. Borgman HP, Bahli B, Heier H, Schewski F (2013) Cloudrise: exploring cloud computing adoption and governance with the TOE framework. In: System Sciences (HICSS), 46th Hawaii International Conference on IEEE., pp 4425-4435

65. Frey S, Hasselbring W (2011) he cloudmig approach: Model-based migration of software systems to cloud-optimized applications. Int J Adv Softw 4, 3(4):342-353

66. Andrikopoulos V, Strauch S, Leymann F (2013) Decision Support for Application Migration to the Cloud. In: Proceedings of CLOSER'13., pp 149-155

67. Juan-Verdejo A, Baars H (2013) Decision support for partially moving applications to the cloud: the example of business intelligence. In: Proceedings of the 2013 international workshop on Hot topics in cloud senvices, ACM., pp 35-42

68. Strauch S, Andrikopoulos V, Karastoynova D (2014) Migrating Enterprise Applications to the Cloud: Methodology and Evaluation. International Journal Big Data Intelligence 1:127-140 